\title{
RELEVANCE AND UNCERTAINTY JOINTLY INFLUENCE REWARD ANTICIPATION AT THE LEVEL OF THE SPN ERP COMPONENT
}

Wioleta Walentowska (1, 2), Katharina Paul (2), Mario Carlo Severo (2), Agnes Moors (2, 3), and Gilles Pourtois (2)

(1) Psychophysiology Laboratory, Institute of Psychology, Jagiellonian University in Krakow, Poland

(2) Cognitive \& Affective Psychophysiology Laboratory, Ghent University, Belgium

(3) Research Group of Quantitative Psychology and Individual Differences, Center for Social and Cultural Psychology, KU Leuven, Belgium

Running head: Relevance and uncertainty influence the SPN component

Corresponding address:

Wioleta Walentowska

Psychophysiology Laboratory

Jagiellonian University in Krakow

Ingardena 6

30-060 Krakow

Poland

e-mail address: wioleta.walentowska@uj.edu.pl

phone number: +48126632408 


\section{Abstract}

The stimulus-preceding negativity (SPN) component reflects the anticipatory phase of reward processing. Its amplitude is usually larger for informative compared to uninformative upcoming stimuli, as well as for uncertain relative to predictable ones. In this study, we sought to assess whether these two effects, when combined together, produced a synergistic effect or rather independent ones on the SPN during performance monitoring. Participants performed a speeded Go/NoGo task while 64-channel EEG was recorded concurrently. We focused on the SPN activity generated in anticipation of feedback, which was either positive (for correct and fast reactions) or negative (for correct but slow responses). Further, the feedback's informativeness about the satisfaction status of goals was alternated across blocks. When uncertainty about the action outcome was low (in conditions where positive feedback was either less or more frequent than negative feedback), the SPN amplitude (measured at fronto-central electrodes) did not vary as a function of feedback's relevance or valence. By comparison, when positive and negative feedback were equiprobable (uncertainty was high), the SPN was more pronounced for relevant compared to irrelevant feedback. Interestingly, in this condition, it was also larger at right fronto-central sites for positive than negative feedback. These ERP results suggest that both factors-relevance and uncertaintycombine and influence reward anticipation at the SPN level.

Keywords: ERP; SPN; reward anticipation; informativeness; relevance; uncertainty

Research highlights:

Informativeness influences performance monitoring and reward processing SPN reflects reward anticipation

SPN is usually larger for informative than uninformative feedback Uncertainty and relevance jointly influence SPN amplitude 


\section{Introduction}

During performance monitoring, both internal (motor) and external (feedback) cues are usually processed and used to adjust behavior when mismatches between goals and actions are detected. Several studies using the event-related potentials (ERP) technique have previously shown that the feedback-related negativity (FRN) component reflects external feedback information processing during performance monitoring. According to the dominant account, the FRN reflects a phasic reward prediction error signal generated by specific fronto-striatal loops (Holroyd and Coles, 2002; Holroyd et al., 2008; Sambrook and Goslin, 2015; Walsh and Anderson, 2012). When the performance or evaluative feedback conveys information about an unexpected mismatch between the hoped-for and the actual outcome, a negativegoing wave is elicited over fronto-central locations, peaking at around $250 \mathrm{~ms}$ post-feedback onset at Fz or FCz sensors. In line with the reward prediction error account, FRN amplitude is larger for unexpected relative to expected events (Hajcak et al., 2007; Pfabigan et al., 2011; von Borries et al., 2013), and for worse-than-expected events, that is, for negative compared to positive performance feedback (Miltner et al., 1997; Nieuwenhuis et al., 2004).

Performance monitoring is a process which is highly dynamic and flexible, seeking to exploit the most informative cue available at a given time and avoiding redundancy (Holroyd and Coles, 2002; Ullsperger et al., 2014). In a situation where the processing of external feedback information is impossible (i.e., feedback information is not available or is perceived as unreliable), the processing of internal (i.e., motor response-based) events prevails and is used to guide the course of performance monitoring. In this situation, the error-related negativity (ERN) component indexing the early, perhaps automatic, detection of response errors based on a swift comparison between the intended and actual motor response is usually elicited at the same fronto-central electrode positions (Falkenstein et al., 1991; Gehring et al., 1993; Ullsperger et al., 2014).

Performance monitoring is not only based on phasic (and reactive) reward prediction error effects upon response execution (ERN) or feedback processing (FRN), but it also usually operates based on 
additional cues that occur after the response but before the outcome, and that are mostly proactive and anticipatory in nature (hence are less phasic and more sustained than the ERN or FRN). Indeed, several earlier ERP studies have identified an ERP component occurring after response execution and during feedback anticipation ${ }^{1}$, called the stimulus-preceding negativity (SPN; Brunia, 1988; Brunia and van Boxtel, 2001; Brunia et al., 2011; Chwilla and Brunia, 1991). The SPN amplitude typically increases from parietal or central to frontal locations, with a right hemispheric dominance usually observed over (pre)frontal areas (F4 vs. F3), consistent with a putative main intracranial generator in the right anterior insular cortex (Brunia, 1988; Brunia et al., 2000, 2011). Even though the SPN has been much less explored in the existing literature than the ERN or FRN components (especially in relation to performance monitoring processes), some studies have already shed light on its putative function. According to the most dominant (motivation-based) model, the SPN is a neurophysiological marker of the anticipation of meaningful information (Brunia and van Boxtel, 2001; Brunia et al., 2011; Kotani et al, 2001, 2003; Masaki et al., 2006, 2010; Ohgami et al., 2004, 2006; van Boxtel and Böcker, 2004). This model predicts a larger and more negative SPN for the anticipation of stimuli that are deemed highly informative compared to ones that carry less information. In line with this, several ERP studies confirmed that the anticipation of stimuli that are informative about rewards and punishments (including monetary gain, evocative photos, or electric shocks) led to a more pronounced SPN component, compared to the anticipation of stimuli considered uninformative or less informative (Kotani et al., 2009; Masaki et al., 2006; van Boxtel and Böcker, 2004).

In performance monitoring research, performance feedback is a good example of a highly informative stimulus. In accordance with this notion, Masaki and colleagues (2010) reported a larger SPN in response to the anticipation of an action-contingent positive feedback (i.e., monetary gain) compared with a reward feedback that was non-contingent on the preceding action. This and other findings have led to the consensus in the psychophysiology literature that the SPN component reflects the anticipation of informative events (Brunia, 1988; Chwilla and Brunia, 1991). It remains currently unclear, however, what

\footnotetext{
${ }^{1}$ This is especially true when the feedback is contingent on behavioral performance and/or motor responses.
} 
"informative" means in this specific framework, especially when it comes to dissecting possible performance monitoring processes at stake during feedback/outcome anticipation.

In previous work (Walentowska et al., 2016), we conceptualized informativeness within the notion of goal relevance (see also Moors, 2007). We distinguished between three separate, but related, meanings of goal relevance: (i) task relevance, which means that a stimulus signals an opportunity to implement goal-directed behavior, which may or may not lead to goal satisfaction; (ii) informativeness, or the degree to which a stimulus is informative about the satisfaction status of pursued goals; and (iii) the impact that a stimulus has on these pursued goals. Feedback stimuli are especially deemed goal relevant in the second and/or third sense. They usually come with a degree of informativeness or trustworthiness (Walentowska et al., 2016), and they can impact on goals to a variable extent (from little to a lot; see also Severo et al., 2017). Note that the type of informativeness at stake here is not simply of any type, but one that conveys information on the satisfaction status of goals. Taking a closer look at previous performance monitoring research studying the role of the SPN component, it seems that informativeness was used there in exactly this second sense. Indeed, in the study of Masaki et al. (2010), performance feedback either did or did not inform the actor about the degree to which his/her action corresponded to an intended action; hence this feedback varied with respect to whether it was informative about the satisfaction status of the goal to engage in the action. Moreover, if the intended action was at the service of higher-order goals, such as the goal to earn money or social status, the performance feedback not only informed the actor about the satisfaction status of the goal to perform well on a particular trial or the task as a whole, but also about the satisfaction status of these higher-order goals (Severo et al., 2017). In this paper, we focus on informativeness as it relates to goal relevance in the second sense. From now on, we refer to this type of informativeness as goal relevance or relevance.

Previous work (Walentowska et al., 2016) already showed that the FRN component (differentiating positive from negative feedback) was elicited when the performance feedback was perceived as goal relevant by the participants but not when it was perceived as irrelevant. Based on these findings, we formulated the prediction that goal-relevant feedback could also elicit a larger SPN 
component than goal-irrelevant feedback. This was the first prediction that we aimed to test in the current study.

Informativeness as it relates to goal relevance is not the only factor used by performance monitoring brain systems during feedback anticipation. Another factor that is very likely to influence this processing stage, but that is rather poorly explored in the existing ERP literature on performance monitoring, is the perceived probability and hence (un)certainty about the upcoming performance feedback. In classical information theory, the notion of uncertainty is linked to the notion of informativeness. As postulated by Shannon (1948), information can be thought of as the resolution of uncertainty. "Information" is a set of possible messages, which should be sent over a noisy channel, and reconstructed by the receiver with low probability of errors, despite the channel noise. The key measure in information theory is "entropy", which quantifies the amount of uncertainty or randomness before an outcome is revealed and hence the degree of information that is carried by the outcome once it is revealed. For example, the coin flip with two equally likely outcomes and hence less uncertainty leads to an outcome with less information (lower entropy) than the roll of a die with six equally likely outcomes and hence more uncertainty. Classical information theory thus entails that the higher the entropy is, the more uncertain the outcome is before it is revealed, and the more information the outcome therefore provides once it is revealed (see also Luce, 2003).

The link between uncertainty and informativeness is also exemplified in animal and human brain studies, in which it has been demonstrated that uncertainty (e.g., the volatility of stimulus-outcome associations) and curiosity play a central role in estimating and learning actions (Bennett et al., 2016; Kid and Hayden, 2015), with a dominant role of the anterior cingulate cortex in decision making and reward processing (Behrens et al., 2007). Interestingly, Bach and Dolan (2012) identified the (anterior) insula as an important limbic structure within a distributed brain network directly involved in computing uncertainty. Given that the (right) anterior insular cortex is thought to be one of the main intracranial generators of the SPN component (Brunia, 1988; Brunia et al., 2011), it is conceivable that uncertainty processing influences the SPN during feedback anticipation. 
Some recent ERP studies have already provided support for the notion that the amplitude of the SPN component is related to uncertainty or unpredictability. Their results showed that the amplitude of the SPN was systematically larger in situations where participants anticipated unpredictable (Catena et al., 2012; Morís et al., 2013) or unexpected reward (Fuentemilla et al., 2013) compared to feedback that was highly predictable or expected. Accordingly, we can derive the prediction that uncertainty should augment the size of the SPN during feedback anticipation. This prediction constituted the second hypothesis tested in the present study.

When positive and negative feedback following action execution are equiprobable, uncertainty about the action outcome is the highest. However, when uncertainty about the action outcome is reduced, the predicted increase in SPN may result not only from low uncertainty but also from changes in motivation in the low reward probability condition, in which negative feedback dominates over positive feedback. In this condition, not only the uncertainty is lower compared to the equiprobable condition, but defensive motivation is also likely to prevail. Because defensive motivation can influence performance monitoring (Koban and Pourtois, 2014), it is unclear in this condition whether (un)certainty or defensive motivation influences reward anticipation at the SPN level. In the high reward probability condition, on the other hand, in which positive feedback dominates over negative feedback, such a change in defensive motivation is unlikely to occur. Thus, to ascertain that uncertainty is one of the key components modulating reward anticipation at the SPN level instead of changes in motivation, it appears important to compare the uncertain condition to both the low and high reward probability conditions. These latter two conditions offer lower uncertainty, albeit in opposite directions (see Figure 1 B). In the current study, we directly addressed this issue and eventually included and compared three levels of reward probability in order to disentangle the uncertainty account from the motivation account at the SPN level.

Previous research on the role of the SPN component has focused either on informativeness in the sense of goal relevance (e.g., Masaki et al., 2010) or on informativeness in the sense of uncertainty reduction (Morís et al., 2013). The main goal of our study was to investigate both information-related factors simultaneously, and to explore their specific contributions to reward anticipation during 
performance monitoring, focusing therefore on the SPN component. To this aim, we devised a factorial design where we could thoroughly explore the interplay of relevance with uncertainty during reward anticipation at the SPN level, and we eventually assessed whether these two variables showed mainly independent effects, or rather a synergistic one during this process (see Figure 1 A). To achieve this, we extracted and analyzed the SPN component from an existing ERP study by Walentowska et al. (2016), in which a speeded Go/NoGo task was used (previously validated extensively across different ERP studies, Aarts and Pourtois, 2010, 2012; Godefroid et al., 2016; Koban et al., 2012; Paul et al., 2017; Pourtois, 2011; Severo et al., 2017; Vocat et al., 2008). In this previous study, both the response-locked (CRN), and stimulus/feedback-locked (FRN and P3) components were carefully analyzed and reported, but not the SPN. Hence the current study reports new results for the SPN that were not part of this earlier publication. Moreover, in this previous report, only two levels of reward probability (i.e., low and intermediate) were considered. In the current study, we compared them to a third level (i.e., high reward probability), for which entirely new EEG data were collected and analyzed.

The added value of using the speeded Go/NoGo task is that participants have to rely on and carefully monitor performance feedback to assess, on a trial-by-trial basis, whether their actions are goal conducive (i.e., correct and fast reactions) or not (i.e., response errors or correct but slow reactions). Moreover, two methodological features are worth mentioning. (i) Because performance is calibrated using an online and adaptive (trial-by-trial and subject-specific) algorithm (see Methods section for details), one can create with this paradigm a situation where feedback is uncertain (i.e., the participant cannot predict easily whether the feedback will be positive or negative before its onset) and two other ones where feedback certainty is substantially increased (i.e., reward probability is clearly low or high), thereby offering an experimental manipulation of (un)certainty across these three conditions. (ii) Orthogonally to this first manipulation, one can also, by means of instructions and a standard cueing technique, devise a situation where it is clear that the performance feedback provided reflects actual performance (i.e., goalrelevant condition), or instead is decoupled from it (goal-irrelevant condition), providing thus a way to manipulate the perceived goal relevance across different blocks. For methodological reasons (see Methods 
section and Walentowska et al., 2016), goal relevance was manipulated across successive blocks and treated as a within-subject variable, while uncertainty (manipulated via reward probability) was manipulated as a between-subjects factor. In light of the evidence reviewed above, we predicted that the amplitude of the SPN component would increase as a function of increased informativeness both in the sense of goal relevance and uncertainty reduction. More specifically, we reckoned that the anticipation of informative performance feedback (because of being relevant and/or uncertainty reducing) would be associated with a larger SPN component compared to uninformative feedback (see Figure 1 A). Importantly, if uncertainty was the main component accounting for amplitude changes at the SPN level (as opposed to motivation), then the low and high reward probability conditions should yield statistically similar effects (i.e., no clear SPN modulation in both cases), compared to the intermediate reward probability condition where the SPN should be the largest because uncertainty was the highest (see Figure $1 \mathrm{~B})$.

[insert Figure 1 about here]

\section{Methods}

\subsection{Participants}

Ninety young healthy adults (university undergraduate students) participated in this study. Thirty (6 men; mean age: $21.9 ; \mathrm{SD}=2.7$ ) were exposed to a version of the speeded Go/NoGo task where reward probability was low (and thus feedback's uncertainty was low), 30 others ( 8 men; mean age: 22.1 ; $\mathrm{SD}=$ 2.3) received a version where reward probability was intermediate (i.e., reward and punishment were equiprobable so that feedback's uncertainty was substantially increased), and another sample of 30 (10 men; mean age: $23.5 ; \mathrm{SD}=2.2$ ) to a high reward probability condition (where feedback's uncertainty was also low). All subjects were right handed and had normal or corrected-to-normal vision. They were free of neurological or psychiatric history and of any psychoactive treatment. Prior to the experiment they gave written informed consent. All subjects participated in the study in exchange of 30 Euro compensation (and 
were not compensated for better performance). The SPN data for the low and intermediate reward probability conditions were extracted from a previously published dataset (with different ERPs in scope; Walentowska et al., 2016). For the high reward probability condition, new EEG data were recorded.

\subsection{Experimental task and design}

A modified version of a well-validated speeded Go/NoGo task (see Aarts and Pourtois, 2010, 2012; Godefroid et al., 2016; Koban et al., 2012; Paul et al., 2017; Pourtois, 2011; Severo et al., 2017; Vocat et al., 2008) was used in the current study (see Figure 1 A for trial structure). Each trial started with the presentation of a black fixation cross lasting for $1000 \mathrm{~ms}$. Then, a black arrow ('cue'), either oriented up or down, was presented. After a variable interval (1000-2000 ms), this black arrow became either green or turquoise, while its in-plane orientation could either remain the same or shift in the opposite orientation. When the black arrow turned green and the orientation remained unchanged ('target'), participants were asked to press a predefined key on the response box as fast as possible with the index finger of their right hand ('Go trials'). However, participants were asked to inhibit their responses when either the arrow became green but flipped orientation, or when the arrow became turquoise and kept its initial orientation ('non-targets' in 'NoGo trials'). In the absence of motor responses, targets and non-targets remained on the screen for $1000 \mathrm{~ms}$. Cues, targets, and non-targets consisted of an arrow, presented in the center of the screen on a white background. After motor responses (correct: 'hits'; incorrect: 'false alarms'), a colored frame was presented around the target $(1000 \mathrm{~ms})$. Next, a response-feedback interval was presented (1000 $\mathrm{ms}$ ), providing the main event of interest for the SPN data analysis (see Figure $1 \mathrm{~A}$ ). This event consisted of the presentation of the colored frame only, and it served to signal to the participant whether the feedback provided was either relevant or irrelevant. The colored frame around the feedback remained on the screen for $1000 \mathrm{~ms}$.

Participants were given positive feedback when they responded both correctly and fast to Go trials ('fast hit'), and when they correctly inhibited responding to NoGo trials ('correct inhibition'). Conversely, they were given negative feedback when the response was correct but too slow ('slow hit'), when they 
responded to NoGo trials ('false alarm'), or when there was no response to Go trials ('omission'). Importantly, we used an online adaptive algorithm to set up a (subject and trial-specific) limit for correct and fast RTs (i.e., response deadline procedure) in Go trials. At the beginning of the experiment, the RT limit was set to $300 \mathrm{~ms}$ (based on previous pilot testing and Vocat et al., 2008). This limit was later adjusted online (i.e., after each trial) as a function of the preceding trial history (thus, as the mean of current and previous RTs). Responses slower than the limit were classified as slow hits, while those faster than the limit were classified as fast hits. The advantage of this algorithm is that uncertainty about current RTs is high throughout the task, which should strongly motivate participants to actively attend to the external feedback stimulus presented after each response to infer whether their actions (during Go trials) were timely (fast hits) or not (slow hits). The response deadline was also updated throughout the whole experimental session in order to avoid habituation or fatigue, and it was originally set up in such a way that correct and fast responding to Go trials was fairly difficult to achieve, reducing uncertainty (i.e., low reward probability - 33\%; see Aarts and Pourtois, 2010; 2012; Dhar and Pourtois, 2011; Dhar et al., 2011; Koban et al., 2010, 2012; Vocat et al., 2008). One group of participants $(\mathrm{n}=30)$ received this specific version of the task.

Another group of participants $(n=30)$ received another version of the exact same speeded Go/NoGo task where the online response deadline to differentiate fast from slow hits was more liberal, increasing in turn reward probability (up to 50\%; intermediate reward probability condition), thereby creating full uncertainty about the feedback. In this version of the task, the RT deadline was adjusted across blocks using specific (predefined) time limits. Specifically, it was set to 300 (in Blocks 1-2), 275 (in Blocks 3-4), and $250 \mathrm{~ms}$ (in Blocks 5-6), yielding in most participants (as established based on extensive piloting, see Walentowska et al., 2016) a balanced number of fast hits and slow hits.

A final group of participants $(n=30)$ was exposed to another version of this speeded Go/NoGo task. Specifically, to create a condition in which uncertainty about the feedback was low, but reward dominated (i.e., high reward probability), the RT deadline was adjusted across blocks using predefined time limits, with 310 (in Blocks 1-2), 300 (in Blocks 3-4), and 290 ms (in Blocks 5-6). These specific 
values were estimated after extensive piloting. Using these settings, participants ended up on average having $66 \%$ of positive feedback (fast hits) and 33\% of negative feedback (slow hits) throughout the experimental session (see Figure 2 A). Accordingly, uncertainty (about the feedback) was manipulated using a between-subjects variable in the current study, being high in the intermediate reward probability condition, and being equally low in both the low and high reward probability conditions.

In this task, feedback following actions on NoGo trials (positive feedback following a correct inhibition or negative feedback following a false alarm) is not informative as participants can evaluate the accuracy of their actions on these trials using internal monitoring and thus motor cues (Koban et al., 2012). Therefore, in this study, we focused primarily on Go trials that were followed either by positive or negative feedback for which conspicuous SPN components were elicited.

Orthogonally to the manipulation of the feedback uncertainty, the goal relevance of the feedback (relevant vs. irrelevant) also varied in a binary way across successive blocks. Hence, goal relevance was manipulated within subjects in this study. Written instructions delivered before the start of each block, as well as a specific colored frame (either blue or magenta) appearing at the response onset (key press), were given to the participants to inform them about the relevance (relevant vs. irrelevant) of the feedback received during this specific block. When the feedback was relevant, it meant that it was genuinely related to the actual speed and the accuracy of the response (thus, participants always received positive feedback after fast hits or correct inhibitions, while they received negative feedback in case of slow hits, false alarms, or omissions). In comparison to that, when being irrelevant, the feedback provided after the response was never related to the actual performance, but randomly drawn from a pre-existing list irrespective of the accuracy and speed of the preceding action performed by the participants. In the low and intermediate reward probability conditions, positive and negative feedbacks were delivered to participants equally often, while in the high reward probability condition irrelevant feedback distribution was matched with relevant context (i.e., throughout the block, participants received $66 \%$ of positive feedbacks and $33 \%$ of negative feedbacks shown in random order). 
To increase monitoring (and relevance), we used emotional faces as evaluative feedback (see Walentowska et al., 2016 for details). To ensure that participants correctly attended to and carefully monitored each and every evaluative feedback, even when they were deemed irrelevant, 'catch trials' were added requiring to perform an additional two-alternative forced choice task based on the valence of the feedback. On average, in $15 \%$ of the trials and in random order, at the offset of the feedback, a question probed participants' ability to categorize the face used as feedback as carrying either a positive or negative emotional expression. For this purpose, participants were instructed to press predefined keys on the response box with the index finger of their right hand. Accuracy, but not speed, was emphasized in this task, and no time limit was imposed.

The experiment consisted of a short training session (32 trials), followed by 6 experimental blocks, each including 56 trials (40 Go and 16 NoGo trials in each block). Go/NoGo trial presentation was randomized within blocks. There were 3 blocks assigned to one of two relevance conditions (relevant vs. irrelevant). Two specific orders were created: R-I-R-I-R-I or I-R-I-R-I-R, with 'R' referring to relevant and 'I' to irrelevant condition. In total, four different versions of the experimental procedure were created, and participants were randomly assigned to one of them, with the mapping between the color of the frame (blue vs. magenta) and relevance (relevant vs. irrelevant) being counterbalanced across participants.

After each block, participants were asked to evaluate how relevant the feedback was during the previous block by means of a Visual Analog Scale (VAS), ranging from -50 (not at all) to +50 (very much). These subjective ratings served as an indirect manipulation check.

Stimulus presentation and response recording were controlled using E-prime software (V2.0., http://www.pstnet.com/products/e-prime/), and all visual stimuli were presented to participants using a 21inch CRT screen.

\subsection{EEG acquisition, ERP data processing, and statistical analyses}

Continuous EEG was acquired at $512 \mathrm{~Hz}$ using a 64-channel (pin-type) Biosemi Active Two system (http://www.biosemi.com), referenced online to the Common ModeSense (CMS)-Driven Right Leg 
(DRL) ground, with the electrodes placed according to the extended International 10-20 EEG system. Horizontal and vertical EOG were monitored by means of 4 electrodes, placed above and below the right eye and on the outer canthi of both eyes. Two additional electrodes were placed at both mastoids.

The SPN was computed offline following a standard procedure of data transformations (Keil et al., 2014): (i) 50-Hz notch filter; (ii) EEG offline referencing using linked mastoids; (iii) -1000/+1000 ms segmentation around the onset of the feedback stimulus; (iv) pre-feedback interval baseline correction (from $-1000 \mathrm{~ms}$ to $-900 \mathrm{~ms})^{2}$; (v) vertical ocular correction for blinks (Gratton et al., 1983); (vi) semiautomatic artifact rejection (trials with motor artifacts were rejected, with a fixed criterion of $\pm 80 \mu \mathrm{V}$ ); (vii) averaging for each response type separately (i.e., fast vs. slow hits); and (viii) low pass digital filtering of the individual average data $(30 \mathrm{~Hz})$. Subsequently, the SPN was defined as the mean voltage within 200 ms prior to the feedback onset (see Catena et al., 2012; Chwilla and Brunia, 1991; Masaki et al., 2010) over left and right frontal (F3, F4), central (C3, C4), and parietal (P3, P4) electrodes, creating in total a set of 6 non-overlapping locations.

The resulting SPN amplitudes were submitted to mixed model ANOVAs with the betweensubjects factor UNCERTAINTY (low/low reward probability vs. high/intermediate reward probability vs. low/high reward probability), and the within-subject factors RELEVANCE (relevant vs. irrelevant), RESPONSE (fast vs. slow hits), SIDE (left vs. right), and LOCATION (frontal vs. central vs. parietal). Significant main or interaction effects were followed up by post-hoc paired $t$-tests.

\section{Results}

\subsection{Behavioral results}

When reward probability was low, and hence uncertainty was low, participants had a larger number of slow hits (approximately 2/3) than fast hits (1/3; see Figure $2 \mathrm{~A}$ ), as confirmed by the main effect of RESPONSE, $F_{(1,29)}=64.85, p<0.0001, \eta_{p}{ }^{2}=0.691$, irrespective of RELEVANCE, $F_{(1,29)}=2.32, p=$

\footnotetext{
${ }^{2}$ We also ran a separate analysis and used the pre-response interval as baseline correction (instead of the postresponse interval), and found that the SPN results remained unchanged.
} 
$0.138, \eta_{p}{ }^{2}=0.074$. When reward probability was intermediate, and hence uncertainty was high, a similar number of fast and slow responses was evidenced (Figure $2 \mathrm{~A}$ ), as revealed by the non-significant main effect of RESPONSE, $F_{(1,29)}=2.01, p=0.101, \eta_{p}{ }^{2}=0.091$, irrespective of RELEVANCE, $F_{(1,29)}=0.25, p$ $=0.621, \eta_{p}{ }^{2}=0.009$. When reward probability was high, and thus the level of uncertainty was low, participants had a significantly larger number of fast hits (approximately 2/3) than slow hits (1/3; see Figure $2 \mathrm{~A}$ ), as confirmed by the main effect of RESPONSE, $F_{(1,29)}=58.61, p<0.0001, \eta_{p}{ }^{2}=0.581$, irrespective of RELEVANCE, $F_{(1,29)}=1.12, p=0.147, \eta_{p}{ }^{2}=0.064$. In none of the conditions did the RESPONSE $x$ RELEVANCE interaction reach significance (all $p s>0.234$ ). A detailed presentation of complementing behavioral results for the low and intermediate reward probability conditions (such as RT speed for each condition, and performance scores for NoGo trials) can be found in Walentowska et al. (2016).

Accuracy for catch trials was high and did not differ as a function of reward probability, and/or relevance. For the low reward probability condition, 93.51\% (SEM =1.23) and 92.61\% (SEM = 1.13) of catch trials were correctly recognized in the relevant and irrelevant blocks, respectively, $t_{(29)}=0.51, p=$ 0.236. In the intermediate reward probability condition, participants correctly categorized $92.69 \%(\mathrm{SEM}=$ $0.97)$ and $92.65 \%(\mathrm{SEM}=0.96)$ of catch trials in the relevant and irrelevant blocks, respectively, $t_{(29)}=$ $0.12, p=0.786$. When reward probability was high, $89.78 \%(\mathrm{SEM}=1.11)$ and $91.44 \%(\mathrm{SEM}=1.23)$ of catch trials were accurately recognized in the relevant and irrelevant blocks, respectively, $t_{(29)}=1.11, p=$ 0.226 .

\subsection{Manipulation checks}

Post-experiment subjective ratings showed that feedback provided in relevant blocks was perceived as more informative than in irrelevant blocks (see Figure 2 B), as confirmed by the significant main effect of RELEVANCE, $F_{(1,87)}=192.67, p<0.0001, \eta_{p}{ }^{2}=0.689$. Interestingly, this effect was reliably modulated by UNCERTAINTY, as revealed by the highly significant RELEVANCE x UNCERTAINTY interaction, $F_{(2,87)}=8.02, p=0.001, \eta^{2}=0.156$. When uncertainty was high, relevant feedback was evaluated as more 
informative than when uncertainty was low (in the low reward probability condition), $t_{(58)}=-3.82, p<$ 0.001, while such a difference was not observed for irrelevant feedback, $t_{(58)}=1.51, p=0.136$. Similarly, participants perceived relevant feedback as more informative in the high uncertainty condition than in the low uncertainty condition (when reward probability was high), $t_{(58)}=3.51, p=0.001$, with no such a difference for irrelevant feedback, $t_{(58)}=0.23, p=0.816$. Importantly, relevant and irrelevant feedback were evaluated comparably in both low uncertainty conditions (with low and high reward probabilities), as confirmed by independent samples $t$-tests: for relevant feedback, $t_{(58)}=-1.12, p=0.268$, and for irrelevant feedback, $t_{(58)}=1.87, p=0.097$.

[insert Figure 2 about here]

\subsection{SPN results}

The ANOVA showed significant main effects of UNCERTAINTY, $F_{(2,87)}=19.81, p<0.0001, \eta_{p}{ }^{2}=0.313$, RELEVANCE, $F_{(1,87)}=13.47, p<0.0001, \eta_{p}{ }^{2}=0.134$, and LOCATION, $F_{(2,174)}=93.09, p<0.0001, \eta_{p}{ }^{2}=$ 0.517. Importantly, UNCERTAINTY interacted significantly with RELEVANCE, $F_{(2,87)}=7.39, p=$ $0.001, \eta_{p}{ }^{2}=0.145$, and SIDE, $F_{(2,87)}=4.35, p=0.016, \eta_{p}{ }^{2}=0.091$. The interaction between SIDE and LOCATION, $F_{(2,174)}=8.47, p<0.0001, \eta_{p}{ }^{2}=0.089$, was also highly significant. All other main or interaction effects were non-significant (all $p s>0.095$ ).

Next, we examined the effect of RELEVANCE at the SPN level separately for each UNCERTAINTY condition. When UNCERTAINTY was low (and reward probability was low; see Figure 3), no systematic change of SPN amplitude across conditions was observed. The ANOVA revealed only a significant main effect of LOCATION, $F_{(2,58)}=27.53, p<0.0001, \eta_{p}{ }^{2}=0.487$, suggesting that the SPN amplitude was not modulated by RELEVANCE in this condition, $t_{(29)}=-1.21, p=0.234$. This main effect of LOCATION indicated a relatively more negative SPN component over central $(M=-0.38 \mu \mathrm{V}$, $\mathrm{SD}=3.47)$ and parietal $(M=-0.63 \mu \mathrm{V}, \mathrm{SD}=2.51)$ than over frontal $(M=3.41 \mu \mathrm{V}, \mathrm{SD}=3.89)$ sites, as confirmed by follow up $t$-tests (frontal vs. central, $t_{(29)}=6.31, p<0.0001$; frontal vs. parietal, $t_{(29)}=6.04, p$ $<0.0001)$. The SPN amplitude did not differ between central and parietal sites, $t_{(29)}=0.45, p=0.655$. 
By contrast, when UNCERTAINTY was high (reward probability level was intermediate; see Figure 4), the SPN amplitude reliably differed as a function of RELEVANCE, with larger amplitudes for relevant than irrelevant feedback. The ANOVA showed significant main effects of RELEVANCE, $F_{(1,29)}=$ 26.15, $p<0.0001, \eta_{p}{ }^{2}=0.474$, SIDE, $F_{(1,29)}=6.83, p=0.014, \eta_{p}{ }^{2}=0.191$, and LOCATION, $F_{(2,58)}=$ 45.91, $p<0.0001, \eta_{p}{ }^{2}=0.613$. Further, the interactions RELEVANCE x SIDE, $F_{(1,29)}=5.52, p=0.026$, $\eta_{p}{ }^{2}=0.161$, RELEVANCE x LOCATION, $F_{(2,58)}=3.61, p=0.033, \eta_{p}{ }^{2}=0.111$, and SIDE x LOCATION, $F_{(2,58)}=10.56, p<0.0001, \eta_{p}{ }^{2}=0.267$ were all significant. Interestingly, the RELEVANCE x RESPONSE interaction was marginally significant in this condition, $F_{(1,29)}=3.72, p=0.064, \eta_{p}{ }^{2}=0.114$.

The significant RELEVANCE x SIDE interaction showed that SPN amplitude was more negative over the right $(M=-7.23 \mu \mathrm{V}, \mathrm{SD}=4.41$, and $-4.41 \mu \mathrm{V}, \mathrm{SD}=3.92$ for the relevant and irrelevant feedback, respectively) than the left hemisphere $(M=-5.98 \mu \mathrm{V}, \mathrm{SD}=5.03$, and $M=-3.69 \mu \mathrm{V}, \mathrm{SD}=3.91$ for the relevant and irrelevant feedback, respectively), especially for relevant feedback. This was confirmed by post-hoc $t$-tests, $t_{(29)}=3.07, p=0.005$ for relevant feedback, and $t_{(29)}=1.88, p=0.091$ for irrelevant feedback.

The RELEVANCE $x$ LOCATION interaction indicated that the SPN amplitude difference between relevant and irrelevant feedback was more pronounced over frontal, $t_{(59)}=-5.03, p<0.0001$, than central, $t_{(59)}=-4.58, p<0.0001$, or parietal sites, $t_{(59)}=-4.74, p<0.0001$.

Lastly, we further explored the trend-significant RELEVANCE x RESPONSE interaction. This interaction effect indicated a numerically larger SPN amplitude following fast hits $(M=-7.13 \mu \mathrm{V}, \mathrm{SD}=$ 5.17) than slow hits $(M=-6.07 \mu \mathrm{V}, \mathrm{SD}=4.69)$ while anticipating relevant feedback, $t_{(29)}=-1.06, p=$ 0.116 , without such a clear asymmetry while anticipating irrelevant feedback $(M=-3.71 \mu \mathrm{V}, \mathrm{SD}=4.11$ for fast hits, and $M=-4.09 \mu \mathrm{V}, \mathrm{SD}=4.11$ for slow hits; $\left.t_{(29)}=1.14, p=0.261\right)$. Further, when focusing on the F4 electrode position only (given our specific prediction about the locus of SPN amplitude modulations as a function of relevance and outcome) for which the SPN amplitude was most sensitive to RELEVANCE (see analyses here above), fast hits (associated with positive feedback) yielded a more 
negative value than slow hits (associated with negative feedback) for relevant, $t_{(29)}=-2.41, p=0.022$ but not for irrelevant feedback, $t_{(29)}=0.14, p=0.886$ (see Figure 4).

Last, when UNCERTAINTY was low (but reward probability was high; see Figure 5), no systematic modulation of SPN amplitude across conditions was noticed. The ANOVA revealed only significant main effects of SIDE, $F_{(1,29)}=4.92, p=0.034, \eta_{p}{ }^{2}=0.145$, and LOCATION, $F_{(2,58)}=24.62, p<$ $0.0001, \eta_{p}{ }^{2}=0.459$. The main effect of SIDE reflected a larger SPN component over right $(M=-5.76 \mu \mathrm{V}$, $\mathrm{SD}=1.33)$ than over left hemisphere $(M=-4.16 \mu \mathrm{V}, \mathrm{SD}=1.27)$. The main effect of LOCATION indicated a more negative SPN component over central $(M=-6.22 \mu \mathrm{V}, \mathrm{SD}=3.21)$ and parietal sites $(M=$ $-7.55 \mu \mathrm{V}, \mathrm{SD}=2.17)$ than over frontal $(M=-1.87 \mu \mathrm{V}, \mathrm{SD}=2.12)$ ones, as confirmed by follow up $t$-tests (frontal vs. central, $t_{(29)}=9.21, p<0.0001$; frontal vs. parietal, $t_{(29)}=11.74, p<0.0001$ ). The SPN amplitude did not differ between central and parietal sites, $t_{(29)}=1.25, p=0.134$.

\section{[insert Figures 3-5 about here]}

\section{Discussion}

Performance monitoring is an utmost important cognitive process that enables self-regulation (Inzlicht et al., 2014). It is based on the swift comparison between goals or intentions and action outcomes, and operates based either on response- (i.e., motor) or feedback-related reward prediction error signals (Holroyd and Coles, 2002; Ullsperger et al., 2014). Yet performance monitoring presumably not only depends on these reactive events, but it can also exploit additional neural signals that are proactive, sustained and anticipatory, and occur during the interval spanning from response execution until feedback delivery. The SPN component is usually elicited during this interval and meets these criteria, especially when participants await the presentation of an informative feedback stimulus (Brunia, 1988; Brunia and van Boxtel, 2001; Brunia et al., 2011), or when an unpredictable outcome is anticipated (Catena et al., 2012; Fuentemilla et al., 2013; Morís et al., 2013). If the feedback is informative, then the SPN elicited prior to it usually grows in size at (right) frontal leads, such as F4 or FC4 electrode positions. 
Based on previous research on SPN, however, it remained unclear which factor connected to informativeness precisely accounted for systematic amplitude modulations of the SPN component during performance monitoring, given that the notion of informativeness was often ill-defined. Building on this, we examined the specific (and possibly joint) contribution of goal relevance and uncertainty to the SPN component in a systematic way. On the one hand, goal relevance has been related to informativeness in a recent study from our group (Walentowska et al., 2016), and the results showed that performance feedback deemed relevant for the participant increases performance monitoring (at the FRN level). On the other hand, classical information theories in psychology (see Shannon, 1948) advocated a long time ago that uncertainty is positively (cor)related with informativeness, and thereby influences a wide range of cognitive processes, including decision making. In this framework, entropy provides a measure of an outcome's uncertainty. More recently, brain imaging studies have shed light on how specific brain regions, such as the ACC and the insula, generate uncertainty estimates in a context-dependent and flexible manner, and in turn alter specific value signals during this process (see also Bach and Dolan, 2012; Behrens et al., 2007; Bennett et al., 2016; Kid and Hayden, 2015).

Our findings confirmed that these two factors can indeed be considered as two sources of information used by dedicated performance monitoring brain processes during feedback (and reward) anticipation. These findings might also explain why the factors studied in previous research (i.e., contingency, expectancy, and reward) led to systematic changes in the amplitude of the SPN during performance monitoring. In addition, our new results clearly confirm that these two factors reliably interacted with one another at the SPN level. Specifically, our results showed that the SPN was larger for relevant than irrelevant feedback, but only when uncertainty about outcome was high, as opposed to low (irrespective of the actual change of reward probability, being either low or high), as demonstrated by a highly significant interaction effect at the statistical level between these two variables. Importantly, because we compared an intermediate reward probability condition (where uncertainty about feedback outcome dominated) to two other ones where uncertainty was substantially reduced and comparable, but going in opposite directions (i.e., low and high reward probability), we could ascertain that SPN amplitude 
modulations during reward anticipation were clearly related to uncertainty, as opposed to (defensive) motivation or valence for example. Below we discuss our findings and their implications for performance monitoring and reward processing in greater detail, while we also hint at some limitations of our study.

Our manipulations of goal relevance and uncertainty turned out to be successful (Figure 2 B). Post-experiment ratings confirmed that participants judged relevant feedback to be overall more informative than irrelevant feedback. Crucially, this effect was significantly larger when the outcome was (highly) uncertain. Noteworthy, the (relevant) feedback was judged equally informative for low and high reward probabilities, these two conditions being evaluated significantly less informative than the intermediate reward probability condition characterized by uncertainty. This pattern of results in turn corroborates the assumption of synergistic effects of relevance and uncertainty during performance monitoring. In short, participants evaluated performance feedback in the elected speeded Go/NoGo task as the most informative when it was both relevant and uncertain, suggesting that these two variables actually combined to shape anticipatory feedback processing at the subject level (see also Figure 1 A and Figure 2 B).

SPN amplitude variations closely mimicked this interaction effect found at the behavioral level. Only if the outcome was highly uncertain (i.e., when a balanced number of positive and negative feedback experienced), then the SPN was eventually larger (more negative) for relevant than irrelevant feedback, especially at the right prefrontal lead (F4) where relevant fast hits (associated with reward/positive feedback) also led to a larger SPN than relevant slow hits (associated with punishment/negative feedback; see Figure 4). This result suggests that uncertainty is probably a pre-requisite to allow for modulation by goal relevance at the SPN level. However, the reverse statement is not true, suggesting that reward processing during the anticipation phase depends more strongly on uncertainty than relevance somehow, or alternatively that relevance is subordinate to uncertainty: If the outcome was relatively certain for the participant (i.e., one outcome - either negative or positive - was associated with a $66 \%$ probability), then the proactive process captured by the SPN allowing to discriminate relevant from irrelevant feedback (and positive from negative) was absent (see Figures 3 and 5), without any clear modulation by feedback 
relevance (or valence). A previous study (see Walentowska et al., 2016), however, did reveal a clear FRN component in the condition of low uncertainty/low reward probability for relevant feedback only, suggesting the presence of a strong reactive component in this condition. Based on this pattern of findings, it is therefore tempting to conclude that uncertainty biases reward-based performance monitoring by increasing "proactive" monitoring in the interval between response onset and feedback delivery, as if the feedback in this condition is potentially more informative for the participant than when uncertainty is reduced. Thus, uncertainty might increase informativeness by boosting or triggering enhanced proactive processes during feedback anticipation (Bennett et al., 2016; Kid and Hayden, 2015). As our new ERP results suggest, this enhanced proactive monitoring created by uncertainty at the SPN level enabled to discriminate relevant from irrelevant feedback (before its delivery). It even enabled to discriminate positive relevant from negative relevant feedback, although this reward anticipation effect was only marginally significant (in the omnibus ANOVA), and limited to the right frontal electrode only (F4) in our study. Note that this latter result is compatible with earlier ERP studies (Brunia, 1988; Brunia et al., 2011; Masaki et al., 2010) that already reported larger SPN effects during performance monitoring or related tasks at the same right compared to left frontal sites.

Although several ERP studies in the past already reported systematic changes of the SPN amplitude as a function of predictability/expectancy (Catena et al., 2012; Fuentemilla et al., 2013; Morís et al., 2013), which can be linked to uncertainty, to the best of our knowledge, our new ERP results are the first to show that besides uncertainty, goal relevance also accounts for them. More specifically, it is likely that amplitude variations found in these earlier studies at the SPN level as a function of expectancy were also partly related to feedback's relevance to some degree, understood as the extent to which it allowed to gauge the actual goal conduciveness of behavior, here corresponding to simple Go/NoGo actions (Walentowska et al., 2016). As a matter of fact, as our new ERP results clearly show, uncertainty alone did not suffice to trigger a reliable SPN component during feedback anticipation; the participant also had to be informed that the upcoming feedback would be goal relevant within this context of uncertainty. In sum, the main contribution of our study consists in clarifying which specific dimensions of information 
may account for SPN amplitude variations during feedback anticipation, and systematically testing the complex interplay between these dimensions at the empirical level, as opposed to referring to "informativeness" broadly defined. In this way, we provide a modern framework that could be valuable for future ERP studies investigating the role of the SPN during performance monitoring. Another main contribution of this work is the confirmation that the SPN is sensitive to uncertainty, as opposed to changes in valence or motivation (see Figure $1 \mathrm{~B}$ ). We observed however as a side note that even though the SPN did not vary with relevance and valence in both the low and high reward probability conditions, its amplitude was generally larger in the high than low reward probability condition (compare Figure 5 and 3). This observation indirectly suggests that feedback anticipation was probably overall reduced when reward probability was low, an effect that might tentatively be explained by a change in defensive motivation between these two opposite conditions (sharing a similar level of uncertainty). Accordingly, future studies should assess the specific contribution of motivation, besides relevance and uncertainty, to amplitude modulations of the SPN during reward anticipation.

A few limitations warrant comment. Following standard practice (Sambrook and Goslin, 2015; Walsh and Anderson, 2012), we mainly manipulated reward probability (i.e., the amount of positive feedback given after fast and correct actions in the present case) to create three different conditions that differed regarding how uncertain the outcome eventually was for the participant throughout the experiment. Although our manipulation checks (see results for subjective ratings, Figure 2 B) confirmed that this was effective (i.e., uncertainty increased perceived relevance of the feedback), we had no more objective measure of feedback uncertainty. Moreover, reward probability was a between-subjects variable in our design. Hence, we cannot formally rule out the possibility that the observed changes at the behavioral/ratings and ERP (SPN) level related to uncertainty actually resulted from uncontrolled group differences. To reduce this risk in our study, all three groups were matched for sample size, age, education, and gender distribution. Future studies may address this limitation by devising experimental designs where uncertainty would be manipulated within-subject (and measured more objectively than via a detour to relevance or informativeness). In a similar vein, future studies might also benefit from 
considering possible changes in state and trait motivation (and affect) with the aim to learn more about motivation-based processes mediating performance monitoring effects (see also Severo et al., 2017). Accordingly, we recommend measuring motivation using standard questionnaires in future studies assessing reward processing at the SPN level. On top of that, investigating the SPN effects as a function of goal relevance in the sense of goal impact (i.e., the third meaning of goal relevance; Severo et al., 2017; Walentowska et al., 2016) could provide some interesting insights. Finally, it might also be fruitful in future ERP studies focused on the SPN component to better model and analyze the local and trial-by-trial predictability of feedback outcome. For example, it may be the case that the amplitude of the SPN not only varies systematically depending on global (blockwise) changes in the perceived goal relevance or reward probability, but that the most recent trial history also dynamically shapes and influences the willingness to acquire and explore new information on the next and subsequent trials, with effects visible at the SPN level. Such approach would require to collect sufficient ERP data depending on the actual sequence of trials (and feedback) encountered, something that we could not have performed in the present study, unfortunately.

To conclude, the present study helped clarify which factors related to informativeness shape reward processing during feedback anticipation. Our new ERP findings suggest that both goal relevance and uncertainty account for amplitude variations at the SPN level during performance monitoring, and produce synergistic effects such that the largest proactive component is observed when the outcome is uncertain, but also goal relevant for the subject. 


\section{References}

Aarts, K., Pourtois, G. 2010. Anxiety not only increases, but also alters early error-monitoring functions. Cogn. Affect. Behav. Ne. 10, 479-492. doi:10.3758/CABN.10.4.479.

Aarts, K., Pourtois, G. 2012. Anxiety disrupts the evaluative component of performance monitoring: An ERP study. Neuropsychologia 50, 1286-1296. doi:10.1016/j.neuropsychologia. 2012.02.012.

Bach, D.R., Hulme, O., Penny, W.D., Dolan, R.J. 2011. The known unknowns: neural representation of second-order uncertainty, and ambiguity. J. Neurosci. 31, 4811-4820. doi: org/10.1523/JNEUROSCI.1452-10.2011.

Bach, D.R., Dolan, R.J. 2012. Knowing how much you don't know: a neural organization of uncertainty estimates. Nat. Rev. Neurosci. 13, 572-586. doi:10.1038/nrn3289.

Behrens, T.E., Woolrich, M.W., Walton, M.E., Rushworth, M.F. 2007. Learning the value of information in an uncertain world. Nat. Neurosci. 10, 1214-1221. doi:10.1038/nn1954.

Bennett, D., Bode, S., Brydevall, M., Warren, H., Murawski, C. 2016. Intrinsic valuation of information in decision making under uncertainty. PLoS Comput. Biol. 12, e1005020. doi: org/10.1371/journal.pcbi.1005020.

Brunia, C.H.M. 1988. Movement and stimulus preceding negativity. Biol. Psychol. 26, 165-178. doi: 10.1016/0301-0511(88)90018-X.

Brunia, C.H.M, de Jong, B.M., van den Berg-Lenssen, M.M., Paans, A.M. 2000. Visual feedback about time estimation is related to a right hemisphere activation measured by PET. Exp. Brain Res. 130, 328-337. doi: 10.1007/s002219900293.

Brunia, C.H.M., van Boxtel, G.J.M. 2001. Wait and see. Int. J. Psychophysiol. 43, 59-75. doi: 10.1016/S0167-8760(01)00179-9.

Brunia, C.H.M., Hackley, S.A., van Boxtel, G.J.M., Kotani, Y., Ohgami, Y. 2011. Waiting to perceive: reward or punishment? Clin. Neurophysiol. 122, 858-868. doi: 10.1016/j.clinph.2010.12.039. 
Chwilla, D.J., Brunia, C.H.M. 1991. Event-related potentials to different feedback stimuli. Psychophysiology 28, 123-132. doi: 10.1111/j.1469-8986.1991.tb00400.x.

Catena, A., Perales, J.C., Megías, A., Cándido, A., Jara, E., Maldonado, A. 2012. The brain network of expectancy and uncertainty processing. Plos ONE 7, e40252. doi: 10.1371/journal.pone.0040252.

Dhar, M., Pourtois, G. 2011. Early error detection is generic, but subsequent adaption to errors is not: evidence from ERPs. Neuropsychologia 49, 1236-1245. doi: 10.1016/j.neuropsychologia.2011.01.006.

Dhar, M., Wiersema, J.R., Pourtois, G. 2011. Cascade of neural events leading from error commission to subsequent awareness revealed using EEG source imaging. PloS ONE 6, e19578. doi: 10.1371/journal.pone.0019578.

Falkenstein, M., Hohnsbein, J., Hoormann, J., Blanke, L. 1991. Effects of crossmodal divided attention on late ERP components. II. Error processing in choice reaction tasks. Electroen. Clin. Neuro. 78, 447-455. doi: 10.1016/0013-4694(91)90062-9.

Fuentemilla, L., Cucurell, D., Marco-Pallarés, J., Guitart-Masip, M., Morís, J., RodríguezFornells, A. 2013. Electrophysiological correlates of anticipating improbable but desired events. NeuroImage, 78, 135-144. doi: 10.1016/j.neuroimage.2013.03.062.

Gehring, W.J., Goss, B., Coles, M.G., Meyer, D.E., Donchin, E. 1993. A neural system for error detection and compensation. Psychol. Sci. 4, 385-390.

Gratton, G., Coles, M.G., Donchin, E. 1983. A new method for off-line removal of ocular artifact. Electroen. Clin. Neuro. 55, 468-484. doi:10.1016/0013-4694(83)90135-9.

Godefroid, E., Pourtois, G., Wiersema, J. R. 2016. Joint effects of sensory feedback and interoceptive awareness on conscious error detection: Evidence from event related brain potentials. Biol. Psychol. 114, 49-60. doi: 10.1016/j.biopsycho.2015.12.005. 
Hajcak, G., Moser, J.S., Holroyd, C.B., Simons, R.F. 2007. It's worse than you thought: The feedback negativity and violations of reward prediction in gambling tasks. Psychophysiology 44, 905-912. doi: 10.1111/j.1469-8986.2007.00567.x.

Holroyd, C.B., Coles, M.G. 2002. The neural basis of human error processing: reinforcement learning, dopamine, and the error-related negativity. Psychol. Rev. 109, 679-709. doi: http://dx.doi.org/10.1037/0033-295X.109.4.679.

Holroyd, C.B., Pakzad-Vaezi, K.L., Krigolson, O.E. 2008. The feedback correct-related positivity: Sensitivity of the event-related brain potential to unexpected positive feedback. Psychophysiology 45, 688-697. doi: 10.1111/j.1469-8986.2008.00668.x.

Inzlicht, M., Schmeichel, B.J., Macrae, C.N. 2014. Why self-control seems (but may not be) limited. Trends Cogn. Sci. 18, 127-133. doi: 10.1016/j.tics.2013.12.009.

Keil, A., Debener, S., Gratton, G., Junghöfer, M., Kappenman, E.S., Luck, S.J., Luu, P., Miller, G. A., Yee, C.M. 2014. Committee report: Publication guidelines and recommendations for studies using electroencephalography and magnetoencephalography. Psychophysiology 51, 1-21. doi: 10.1111/psyp.12147.

Kidd, C., Hayden, B.Y. 2015. The psychology and neuroscience of curiosity. Neuron 88, 449460. doi: org/10.1016/j.neuron.2015.09.010.

Koban, L., Pourtois, G., Vocat, R., Vuilleumier, P. 2010. When your errors make me lose or win: Event-related potentials to observed errors of cooperators and competitors. Soc. Neurosci. 5, 360-374. doi: 10.1080/17470911003651547.

Koban, L., Pourtois, G., Bediou, B., Vuilleumier, P. 2012. Effects of social context and predictive relevance on action outcome monitoring. Cogn. Affect. Behav. Ne. 12, 460-478. doi: 10.3758/s13415012-0091-0.

Koban, L., Pourtois, G. 2014. Brain systems underlying the affective and social monitoring of actions: An integrative review. Neurosci. Biobehav. R. 46, 71-84. doi: org/10.1016/j.neubiorev.2014.02.014. 
Kotani, Y., Hiraku, S., Suda, K., Aihara, Y. 2001. Effect of positive and negative emotion on stimulus-preceding negativity prior to feedback stimuli. Psychophysiology 38, 873-878. doi: 10.1111/1469-8986.3860873.

Kotani, Y., Kishida, S., Hiraku, S., Suda, K., Ishii, M., Aihara, Y. 2003. Effects of information and reward on stimulus-preceding negativity prior to feedback stimuli. Psychophysiology 40, 818-826. doi: 10.1111/1469-8986.00082.

Kotani, Y., Ohgami, Y., Kuramoto, Y., Tsukamoto, T., Inoue, Y., Aihara, Y. (2009). The role of the right anterior insular cortex in the right hemisphere preponderance of stimulus-preceding negativity (SPN): an fMRI study. Neurosci. Lett. 450, 75-79. doi: org/10.1016/j.neulet.2008.11.032.

Luce, R.D. 2003. Whatever happened to information theory in psychology? Rev. Gen. Psychol. 7, 183. doi: /10.1037/1089-2680.7.2.183.

Masaki, H., Takeuchi, S., Gehring, W.J., Takasawa, N., Yamazaki, K. 2006. Affectivemotivational influences on feedback-related ERPs in a gambling task. Brain Res. 1105, 110-121. doi: 10.1016/j.brainres.2006.01.022.

Masaki, H., Yamazaki, K., Hackley, S.A. 2010. Stimulus-preceding negativity is modulated by action-outcome contingency. NeuroReport 21, 277-281. doi: 10.1097/WNR.0b013e3283360bc3.

Miltner, W.H., Braun, C.H., Coles, M.G. 1997. Event-related brain potentials following incorrect feedback in a time-estimation task: Evidence for a "generic" neural system for error detection. J. Cogn. Neurosci. 9, 788-798 doi:10.1162/jocn.1997.9.6.788.

Moors, A. 2007. Can cognitive methods be used to study the unique aspect of emotion: An appraisal theorist's answer. Cognition Emotion 21, 1238-1269. doi:10.1080/02699930701438061.

Nieuwenhuis, S., Holroyd, C.B., Mol, N., Coles, M.G. 2004. Reinforcement-related brain potentials from medial frontal cortex: origins and functional significance. Neurosci. Biobehav. R. 28, 441448. doi:10.1016/j.neubiorev.2004.05.003.

Morís, J., Luque, D., Rodríguez-Fornells, A. 2013. Learning-induced modulations of the stimuluspreceding negativity. Psychophysiology 50, 931-939. doi: 10.1111/psyp.12073. 
Ohgami, Y., Kotani, Y., Hiraku, S., Aihara, Y., Ishii, M. 2004. Effects of reward and stimulus modality on stimulus-preceding negativity. Psychophysiology 41, 729-738. doi: 10.1111/j.14698986.2004.00203.x.

Ohgami, Y., Kotani, Y., Tsukamoto, T., Omura, K., Inoue, Y., Aihara, Y., Nakayama, M. 2006. Effects of monetary reward and punishment on stimulus-preceding negativity. Psychophysiology 43, 227 236. doi: 10.1111/j.1469-8986.2006.00396.x.

Paul, K., Walentowska, W., Bakic, J., Dondaine, T., Pourtois, G. 2017. Modulatory effects of happy mood on performance monitoring: Insights from error-related brain potentials. Cogn. Affect. Behav. Ne. 17, 106-123. doi: 10.3758/s13415-016-0466-8.

Pfabigan, D.M., Alexopoulos, J., Bauer, H., Lamm, C., Sailer, U. 2011. All about the money external performance monitoring is affected by monetary, but not by socially conveyed feedback cues in more antisocial individuals. Front. Hum. Neurosci. 5. doi: 10.3389/fnhum.2011.00100.

Pourtois, G. 2011. Early error detection predicted by reduced pre-response control process: An ERP topographic mapping study. Brain Topogr. 23, 403-422. doi:10.1007/s10548-010-0159-5.

Sambrook, T.D., Goslin, J. 2015. A neural reward prediction error revealed by a meta-analysis of ERPs using great grand averages. Psychol. Bull. 141, 213-235. doi: 10.1037/bul0000006.

Severo, M.C., Walentowska, W., Moors, A. Pourtois, G. 2017. Goal impact influences the evaluative component of performance monitoring: Evidence from ERPs. Biol. Psychol. 129, 90-102. doi: org/10.1016/j.biopsycho.2017.08.052.

Shannon, C.E. 1948. A mathematical theory of communication (parts I and II). Bell Syst. Tech. J. 27, 379-423. doi: 10.1002/j.1538-7305.1948.tb01338.x.

Ullsperger, M., Fischer, A.G., Nigbur, R., Endrass, T. 2014. Neural mechanisms and temporal dynamics of performance monitoring. Trends Cogn. Sci. 18, 259-267. doi:10.1016/j.tics.2014.02.009.

van Boxtel, G.J., Böcker, K.B. 2004. Cortical Measures of Anticipation. J. Psychophysiol. 18, 6176. doi: $10.1027 / 0269-8803.18 .23 .61$. 
von Borries, A.K.L., Verkes, R.J., Bulten, B.H., Cools, R., de Bruijn, E.R.A. 2013. Feedbackrelated negativity codes outcome valence, but not outcome expectancy, during reversal learning. Cogn. Affect. Behav. Ne. 13, 737-746. doi: 10.3758/s13415-013-0150-1.

Vocat, R., Pourtois, G., Vuilleumier, P. 2008. Unavoidable errors: a spatio-temporal analysis of time-course and neural sources of evoked potentials associated with error processing in a speeded task. Neuropsychologia 46, 2545-2555. doi:10.1016/j.neuropsychologia.2008.04.006.

Walentowska, W., Moors, A., Paul, K., Pourtois, G. 2016. Goal relevance influences performance monitoring at the level of the FRN and P3 components. Psychophysiology 53, 1020-1033. doi: 10.1111/psyp.12651.

Walsh, M.M., Anderson, J.R. 2012. Learning from experience: event-related potential correlates of reward processing, neural adaptation, and behavioral choice. Neurosci. Biobehav. R. 36, 1870-1884. doi:10.1016/j.neubiorev.2012.05.008. 


\section{Author Note}

This study is supported by funding from the National Science Centre of Poland (NCN grant 2015/19/B/HS6/01259). GP is supported by the Belgian Science Policy, Interuniversity Attraction Poles program (P7/11) and by a Concerted Research Action Grant from Ghent University. He is the recipient of an (2015) independent investigator grant awarded by the NARSAD foundation. GP and AM are also supported by a research grant from the Research Foundation Flanders (FWO grant G024716N). PhD scholarship of KP is funded by the Research Foundation Flanders (FWO).

Conflict of interest: none declared.

Correspondence should be sent to: Wioleta Walentowska, Psychophysiology Laboratory, Jagiellonian University in Krakow, Ingardena 6, 30-060 Krakow, Poland. 
A)

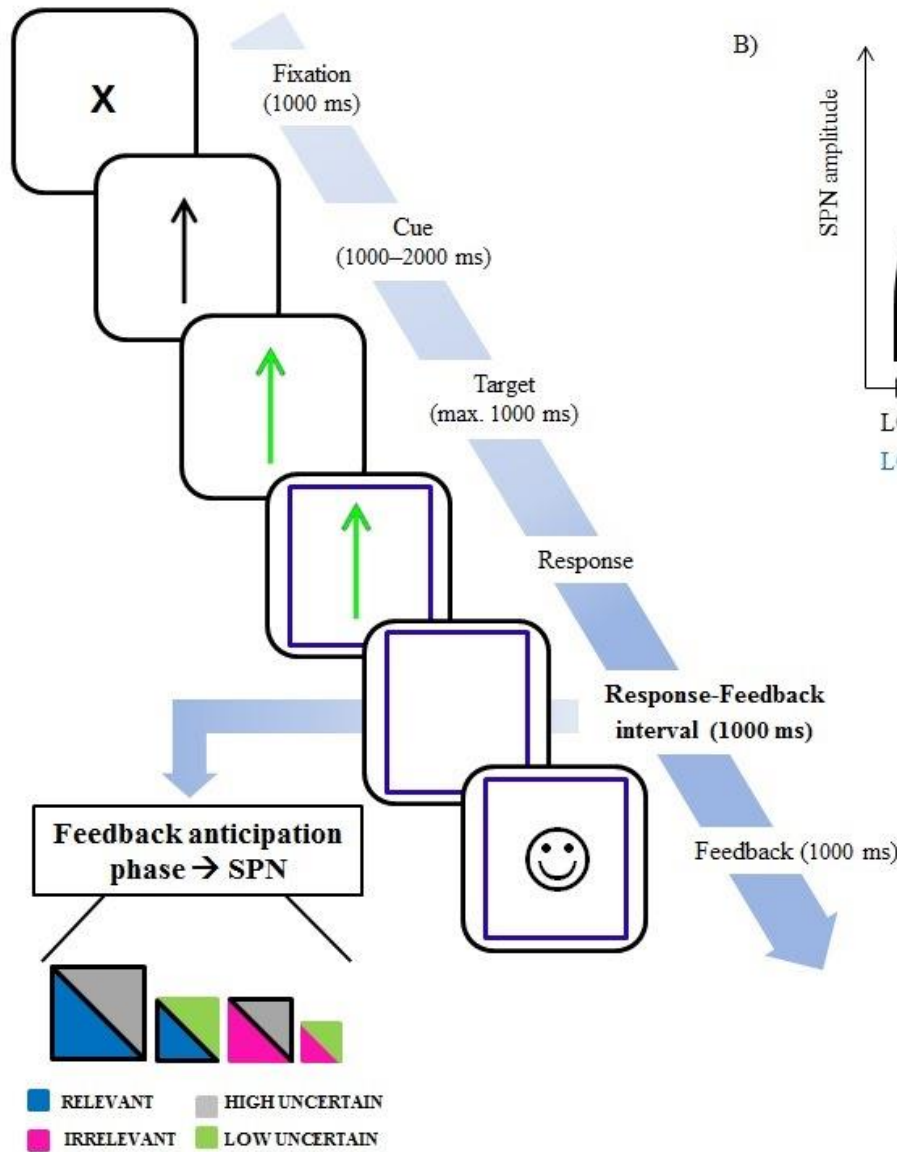

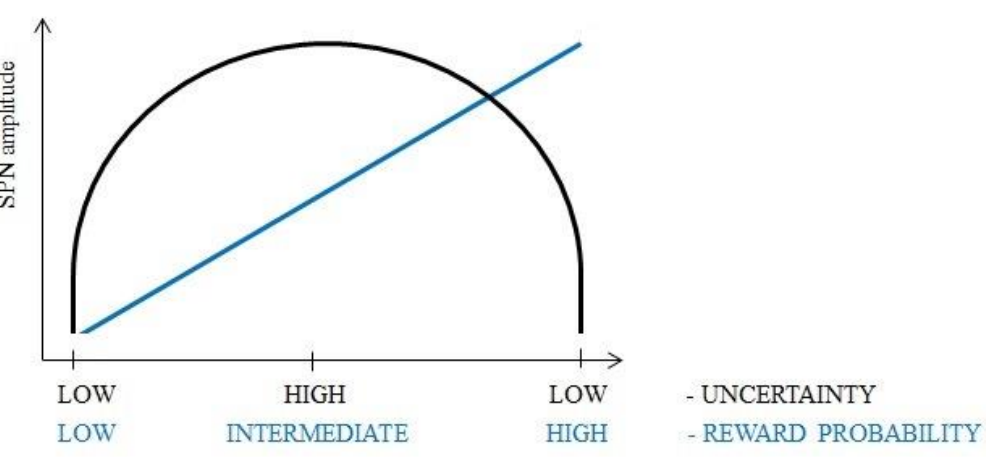

Figure 1. (A) Trial structure. At response onset (speeded Go/NoGo decision), a colored frame appeared around the target, signaling the response-feedback relation (relevant context) or lack thereof (irrelevant context). The frame stayed on screen until feedback offset, creating the main event of interest (feedback anticipation phase), and giving rise to the SPN component. One thousand ms elapsed after response onset until feedback delivery. The SPN component was measured as a mean ERP activity (at several electrode positions) during the $200 \mathrm{~ms}$ interval prior to the feedback onset. If feedback was informative (and carried reward-related information), expectations about reward grew prior to feedback onset, and enlarged the SPN component. In our study, feedback was deemed informative when both criteria of informativeness (relevance and uncertainty, marked here with a black frame) were met (as depicted by the large size of the colorful square; see lower left panel). Feedback was presumably less informative when only one of them was fulfilled (corresponding to the medium-sized square). In case none of them was met, feedback was 
considered as uninformative (the smallest square). (B) Theoretically, the SPN amplitude could vary depending either on feedback's uncertainty (inverted u-shape function) or valence/motivation (linear effect). If the SPN amplitude reflects uncertainty, then it is expected to be the largest when the outcome is uncertain and the lowest when it is certain, irrespective of the dominating valence. In contrast, if the SPN amplitude is sensitive to valence and motivation, then it should encode changes in reward probability, with the smallest SPN for low reward probability, and the largest for high reward probability. 
A)
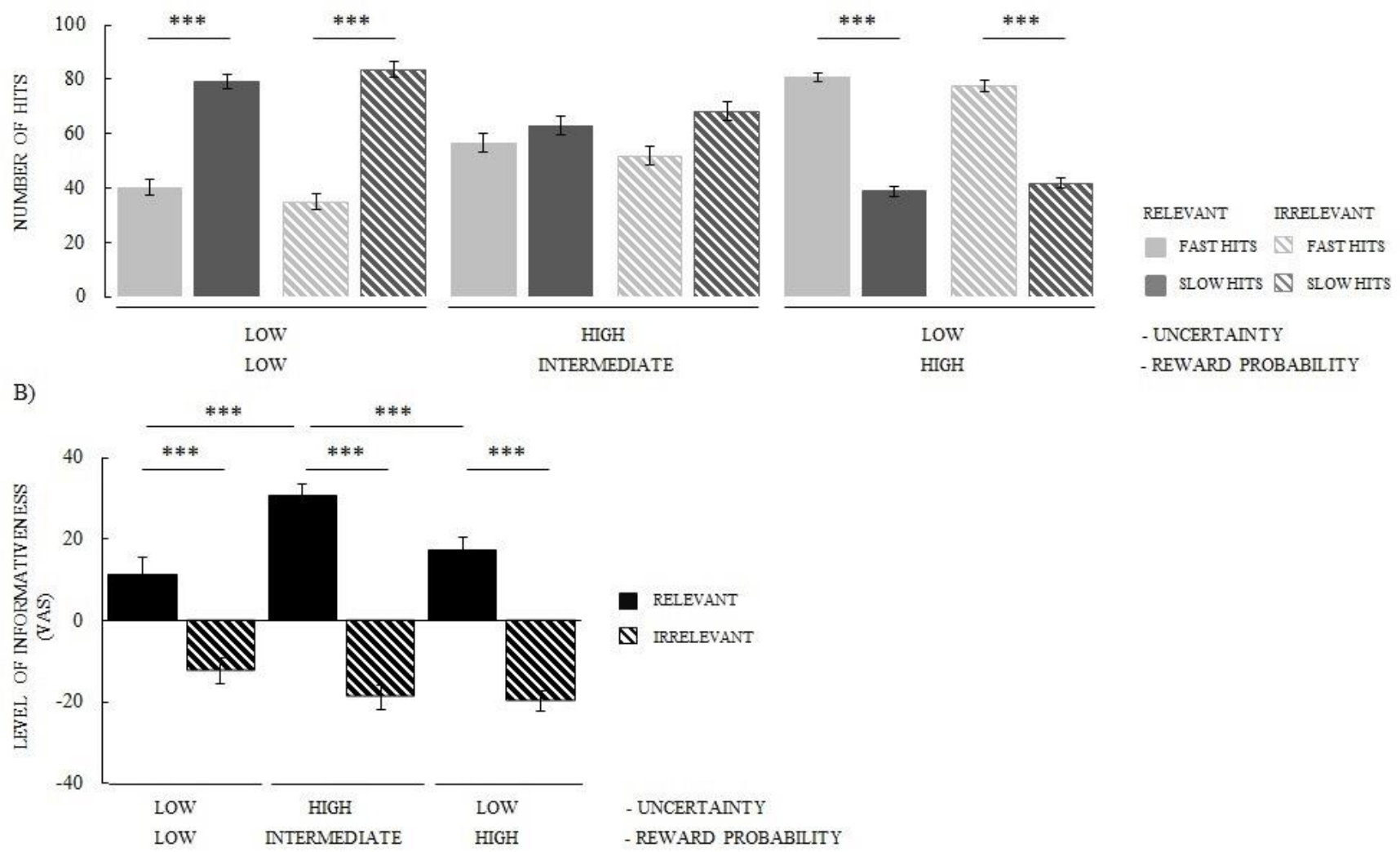

Figure 2. (A) Mean number of accurate and timely (fast hits) and accurate but slow (slow hits) responses achieved by participants in our study, confirming that the manipulation was successful. At low uncertainty, reward probability was either low or high, irrespective of relevance. In contrast, at high uncertainty, positive and negative feedback were equiprobable, again, irrespective of relevance. (B) Manipulation checks of feedback's informativeness (based on the VAS scale). Relevant feedback was evaluated as more informative than irrelevant feedback. Interestingly, when uncertainty was high, this effect was statistically stronger than when uncertainty was low (irrespective of low or high reward probability). In other words, participants judged the feedback as the most informative when it was both uncertain and relevant, corroborating that these two variables combined to shape informativeness at the subject level. Note that the scores can vary from -50 (not informative at all) to +50 (very informative). Error bars represent standard errors of the mean (SEM). $* * * p<0.001$. 
A)

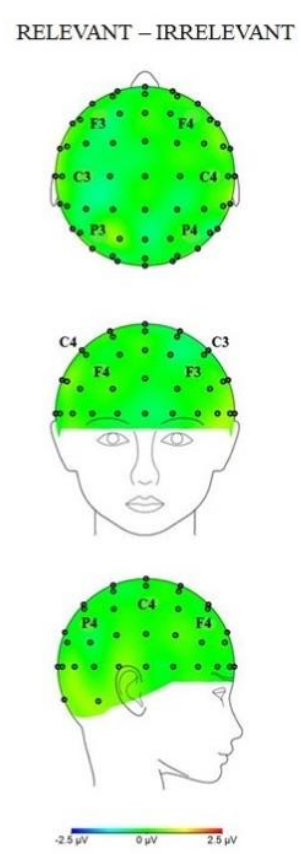

B)

F3

C3

P3

$\mathbf{R}$

$\mathbf{R}$
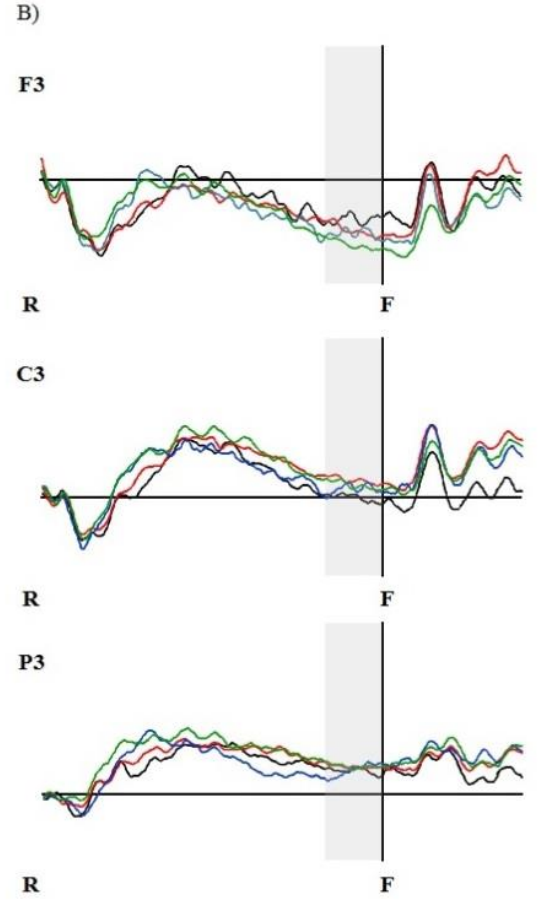

F4

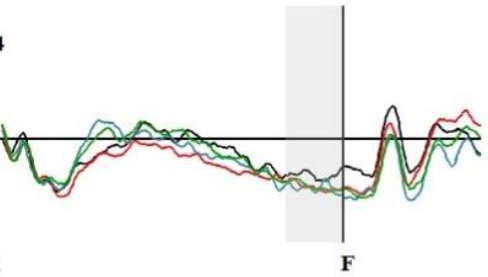

$\mathbf{R}$

C4

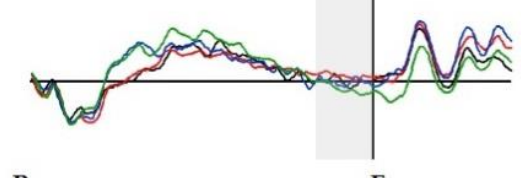

$\mathbf{R}$

P4

$\mathbf{R}$

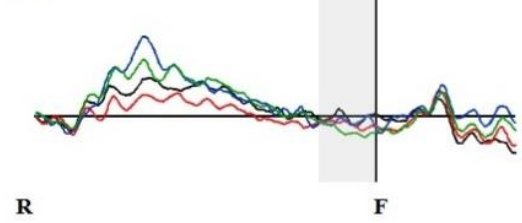

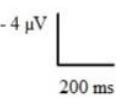

RELEVANT

- FAST HITS - SLOW HIT IRRELEVANT FAST HITS - SLOW HITS

Figure 3. (A) Topographical maps (horizontal, frontal and lateral views) of SPN results (mean ERP activity computed during $200 \mathrm{~ms}$ prior to the feedback onset). Each map shows an ERP difference wave where irrelevant feedback is subtracted from relevant feedback. (B) Feedback-preceding grand average ERP waveforms recorded from F3, F4, C3, C4, P3, and P4 electrodes for the SPN interval. When uncertainty was low, the SPN component was modulated neither by the relevance nor the response type. Note that R stands for response onset, and F for feedback onset. The time window of interest (-200/0 ms) is highlighted, and negativity is plotted upwards. 


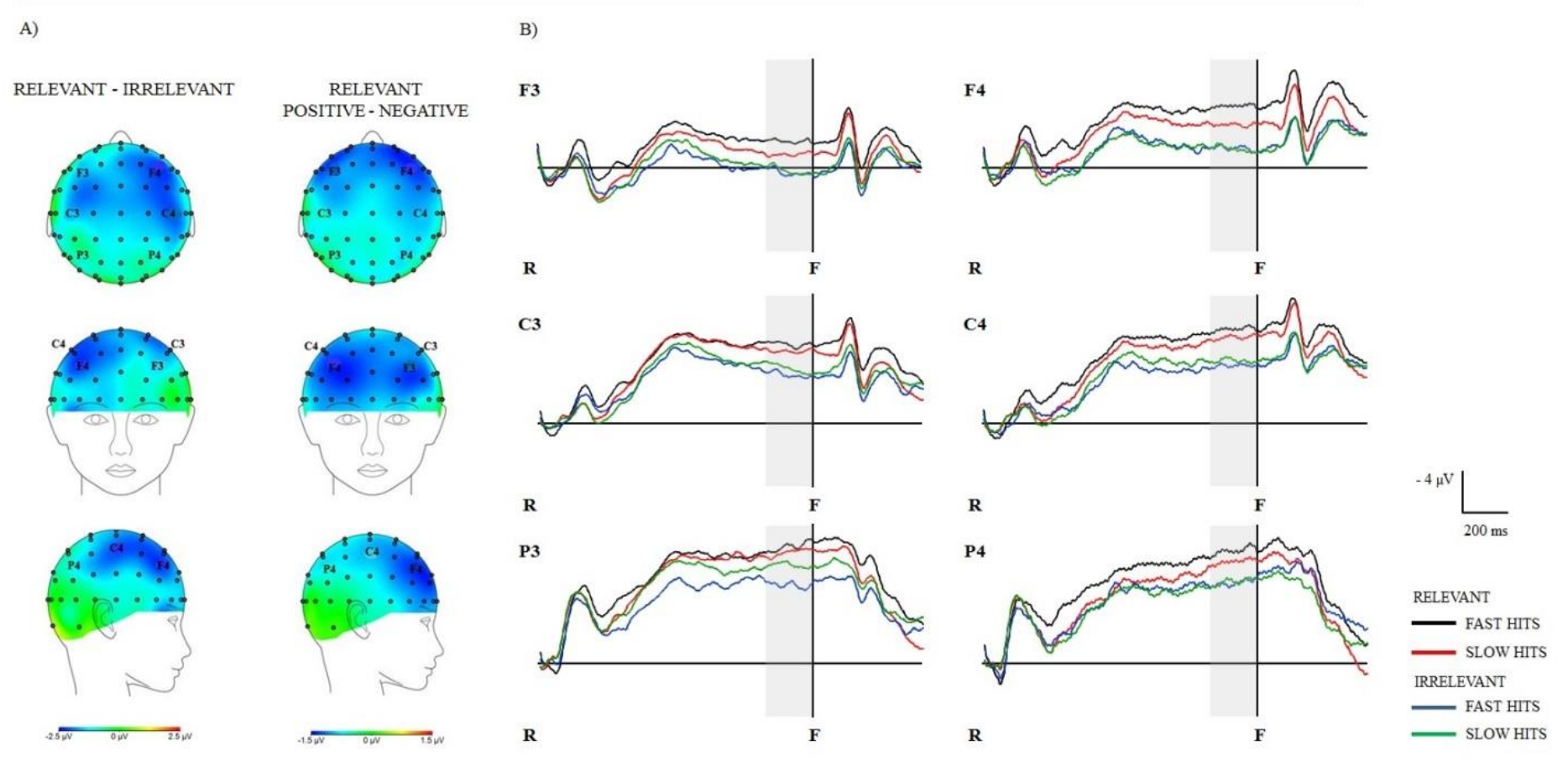

Figure 4. (A) Topographical maps (horizontal, frontal and lateral views) of SPN results (mean ERP activity computed during $200 \mathrm{~ms}$ prior to the feedback onset). Each map shows an ERP difference wave where irrelevant feedback is subtracted from relevant feedback (left column), while the right column shows the SPN valence effect (where negative feedback is subtracted from positive feedback). (B) Feedback-preceding grand average ERP waveforms, recorded from F3, F4, C3, C4, P3, and P4 electrode positions. Exclusively in this condition, the SPN component was larger for relevant than irrelevant feedback. Moreover, at the F4 position, it was also larger for positive than negative relevant feedback, suggesting reward anticipation when the feedback was both uncertain and relevant. Note that $\mathrm{R}$ stands for response onset, and F for feedback onset. The time window of interest $(-200 / 0 \mathrm{~ms})$ is highlighted, and negativity is plotted upwards. 
A)

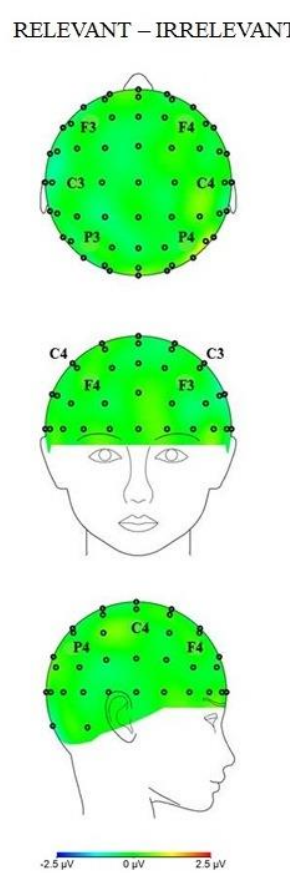

B)

F3

$\mathbf{R}$

C3

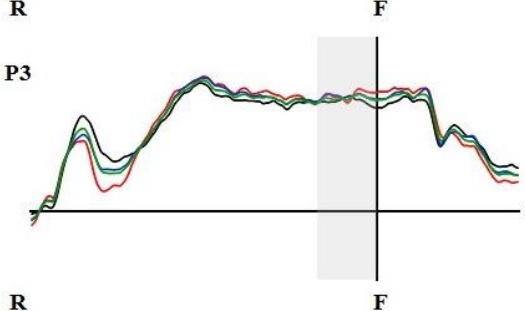

F4

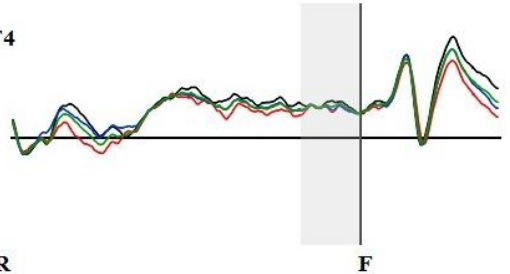

$\mathrm{C} 4$

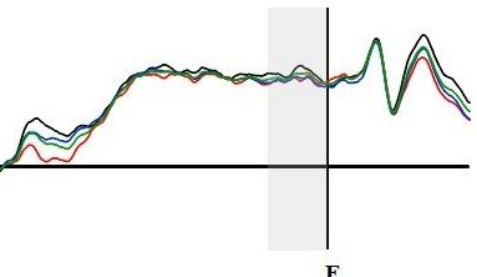

$\mathbf{R}$

P4

R

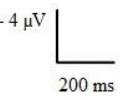

RELEVANT

- FAST HITS IRRELEVANT — FAST HITS

Figure 5. (A) Topographical maps (horizontal, frontal and lateral views) of the SPN results (mean ERP activity computed during $200 \mathrm{~ms}$ prior to the feedback onset). Each map shows an ERP difference wave where irrelevant feedback is subtracted from relevant feedback. (B) Feedback-preceding grand average ERP waveforms recorded from F3, F4, C3, C4, P3, and P4 electrodes for the SPN interval. The SPN component was not modulated by the relevance nor the response type when uncertainty about the action outcome was low. Note that $\mathrm{R}$ stands for response onset, and $\mathrm{F}$ for feedback onset. The time window of interest (-200/0 ms) is highlighted, and negativity is plotted upwards. 